\section{Plasmocytes tumoraux et autophagie}

\section{Une relation ambiguë}

Laurence Lamy
National cancer institute, National institutes of health, metabolism branch, 9000 Rockville Pike, Bethesda, Maryland 20892, États-Unis.

lamy.laurence@gmail.com
> L'autophagie est un mécanisme ancien, conservé de la levure aux mammifères, qui contrôle la dégradation d'une partie des protéines cytoplasmiques dans le but de produire de l'énergie, recycler des organites cellulaires défectueux, éliminer des pathogènes ou rétablir l'homéostasie de la cellule. Dans un premier temps, une double membrane se constitue, s'allonge et séquestre progressivement une partie du cytoplasme, constituant un autophagosome. Cette vacuole fusionne ensuite avec un lysosome pour former un autophagolysosome. Le matériel séquestré dans la lumière autophagosomale est ensuite dégradé en acides gras et en acides aminés pouvant être réutilisés par la cellule. L'autophagie, qui est activée en réponse à une situation de stress comme une déplétion en nutriments ou en facteurs de croissance, permet à la cellule de s'adapter et de survivre jusqu'au rétablissement des conditions d'homéostasie cellulaire.

Lorsque les conditions sont favorables, un niveau basal d'autophagie participe au maintien de l'homéostasie cellulaire. Cette autophagie dite constitutive est particulièrement importante pour les cellules différenciées qui n'ont plus les moyens de diluer les organites détériorés et les agrégats protéiques en se divisant (pour revue, voir $[1,2]$ ).

\section{La survie des plasmocytes normaux et tumoraux est dépendante de l'autophagie}

Les plasmocytes sont l'exemple même de cellules différenciées dont la survie est dépendante de l'autophagie constitutive. Ces cellules représentent le stade final de différenciation des lymphocytes $B$ et sont caractérisées par la production et la sécrétion de grandes quantités d'immunoglobulines spécifiques des antigènes rencontrés. Les plasmocytes ne se divisent plus, mais ils peuvent survivre de nombreuses années dans le microenvironnement de la moelle osseuse. L'importance de l'autophagie dans la survie des plasmocytes a été confirmée récemment chez des souris déficientes pour la protéine ATG5 (autophagy protein 5), un composant de la machinerie autophagique [3]. Chez ces souris, les plasmocytes sont incapables de réguler la taille de leur réticulum endoplasmique et leur production d'immunoglobulines. Cela conduit à la déplétion des réserves d'ATP et à l'augmentation du stress cellulaire, provoquant la mort des cellules.

Le myélome multiple est une hémopathie caractérisée par la présence de plasmocytes tumoraux qui, par leur pouvoir prolifératif, aboutissent à l'envahissement de la moelle osseuse. Comme les plasmocytes normaux, les plasmocytes tumoraux doivent faire face à un stress métabolique constant, généré par l'accumulation et l'agrégation dans leur cytoplasme de grandes quantités d'immunoglobulines (Figure 1). Pour éliminer ces agrégats toxiques, les plasmocytes ont recours à plusieurs systèmes complémentaires : des molécules chaperons, pour corriger le défaut de repliement des protéines néosynthétisées; le protéasome et l'autophagie pour leur dégradation, l'autophagie étant seule capable de dégrader les agrégats protéiques trop volumineux. Ainsi, des études montrent la surexpression des molécules chaperons (en particulier Hsp90, heat shock protein 90) dans les plasmocytes de myélome multiple [4], une activité accrue du protéasome [5] et une autophagie constitutive relativement élevée [6]. Ces observations ont été exploitées sur un plan thérapeutique: des inhibiteurs du protéasome, tels que le Velcade (Bortezomib) et le Kyprolis (Carfilzomib), sont couramment utilisés dans le traitement des patients atteints de myélome multiple, et des analogues de la geldanamycine, un inhibiteur d'Hsp90, sont en phase II d'études cliniques [7]. Enfin, la chloroquine et la 3-méthyladénine (3-MA) induisent la mort cellulaire des plasmocytes tumoraux via l'inhibition de la voie autophagique [6].

La caspase-10 limite l'autophagie et contrôle le stress métabolique dans le myélome multiple

Lors d'un criblage génétique basé sur une approche d'ARN interférence appliquée à différentes lignées de myélomes multiples [8], nous avons identifié la caspase-10 comme une molécule indispensable à la survie des plasmocytes tumoraux [9]. Cette découverte est étonnante dans la mesure où la caspase-10 est surtout connue pour son rôle clé dans la régulation de l'apoptose en réponse à l'activation des récepteurs 


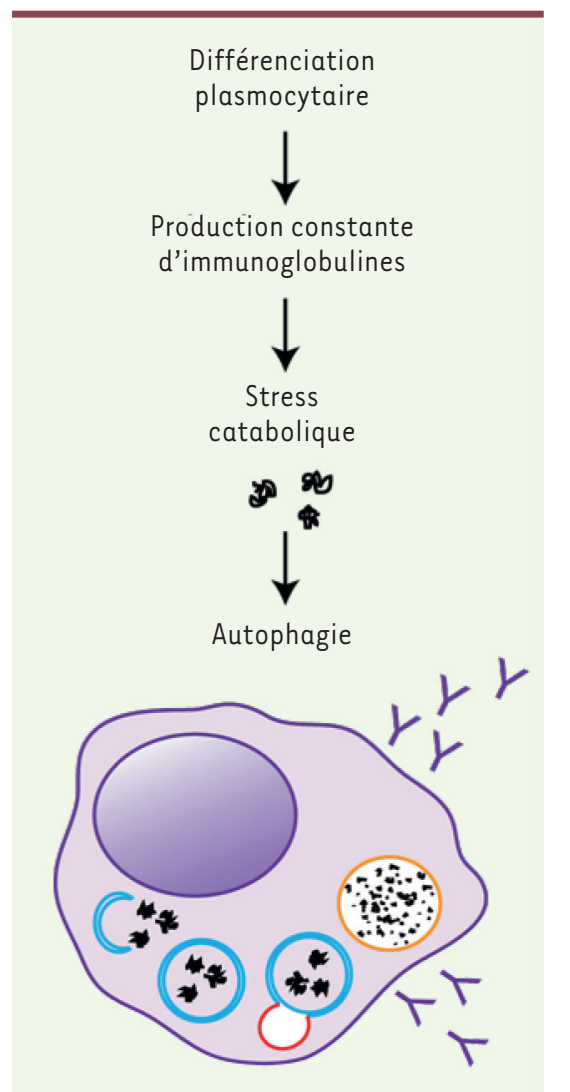

Survie des plasmocytes normaux et tumoraux

Figure 1. L'autophagie constitutive régule le stress métabolique des cellules plasmatiques normales et tumorales. Les plasmocytes sont caractérisés par une production abondante d'immunoglobulines. L'une des conséquences est l'accumulation toxique de protéines mal repliées dans le cytoplasme. L'autophagie est l'un des mécanismes qui permet aux plasmocytes d'éliminer ces agrégats protéiques et de réduire le niveau de stress métabolique.

de mort. Notre étude montre que la caspase-10 est partiellement active dans les plasmocytes tumoraux multiples et forme un complexe avec la forme longue de cFLIP (cFLIP , cellular FLICE-like inhibitory protein). Ce complexe peut être inhibé par le AEVD-OPH, un inhibiteur de la caspase-10, qui induit une vacuolisation des cellules et leur mort. Des clichés de microscopie électronique montrent des vacuoles à double membrane caractéristiques des autophagosomes, ainsi que des autophagolysosomes dont le nombre et la taille augmentent dans les jours qui suivent l'inhibition du complexe caspase-10-cFLIP . Le noyau cellulaire, en revanche, reste intact, et la mort des cellules ne s'accompagne ni de l'externalisation de la phosphatidylsérine, ni de l'activation de la caspase-3, indiquant que l'apoptose n'est pas impliquée dans cette mort cellulaire. D'autre part, l'inhibition de la caspase-10 entraîne le clivage la protéine LC3 (light chain 3) et sa relocalisation à la membrane des autophagosomes, indiquant clairement qu'un processus autophagique est en cours. De plus, l'inhibition de deux molécules clé du processus autophagique, la bécline-1 et ATG5, protège les cellules de la mort produite par l'inhibition de la caspase-10, confirmant ainsi que l'autophagie induite dans les plasmocytes tumoraux n'est pas un mécanisme de protection de la cellule qui n'aurait pas abouti, mais bien le mécanisme par lequel la cellule est éliminée. II est important de noter que l'inhibition de la bécline-l et le traitement des cellules par la 3-MA se sont avérés toxiques dans notre étude, confirmant l'importance de l'autophagie en tant que mécanisme constitutif de survie des cellules tumorales de myélome multiple.

Par ailleurs nous avons identifié BCLAFI (BCL2-associated transcription factor 1) comme étant un substrat de la caspase-10 et montré qu'il est clivé de façon constitutive dans les myélomes multiples. La surexpression d'une forme inductible de BCLAFl entraîne le clivage de LC3 et l'accumulation de vacuoles autophagiques, comme le fait l'inhibition de la caspase-10, suggérant que l'accumulation de BCLAFl contribue au phénotype autophagique. BCLAFl a été identifiée initialement comme une protéine s'associant à différents membres anti-apoptotiques de la famille BCL2 [10]. Outre leur rôle dans l'inhibition de l'apoptose, plusieurs membres de cette famille peuvent réguler l'autophagie en s'associant à la bécline-1 à la surface du réticulum endoplasmique [11]. Nous montrons qu'en stabilisant BCLAFl via l'inhibition du complexe caspase-10-cFLIP $P_{L}$, l'association de BCLAFl à BCL2 est amplifiée, ce qui a pour conséquence de libérer la bécline-l et d'enclencher l'autophagie (Figure 2).

Cibler la caspase-10 : une approche thérapeutique dans le myélome multiple?

Le myélome multiple reste une maladie incurable, complexe en raison d'une grande variabilité génétique. II existe donc un réel besoin de développer des traitements ciblant des mécanismes de survie cellulaire partagés par une grande majorité de ces cellules cancéreuses. Notre étude montre que les cellules tumorales dans le myélome multiple, quelles que soient leurs caractéristiques génétiques, ont un rapport ambivalent avec l'autophagie. Une régulation trop stricte de cette dernière les met en danger face à l'accumulation de protéines toxiques et à la déplétion des ressources énergétiques. À l'opposé, la perte de mécanismes de contrôle de l'autophagie, comme nous le montrons pour le complexe caspase-10-cFLIP, active de façon excessive le phénomène d'autophagie, ce qui finit par détruire la cellule. De façon intéressante, notre étude montre que la caspase-10 est indispensable à la survie cellulaire dans tous les cas de myélome testés. Son expression est induite au niveau transcriptionnel par le facteur de transcription IRF4 (interferon regulatory factor 4 ), un des acteurs principaux de la différenciation plasmocytaire. Il est donc probable que l'expression de la caspase-10 est liée au programme génétique des plasmocytes et que son induction représente un processus adaptatif crucial pour la survie de ces cellules. Le développement de drogues privilégiant une 


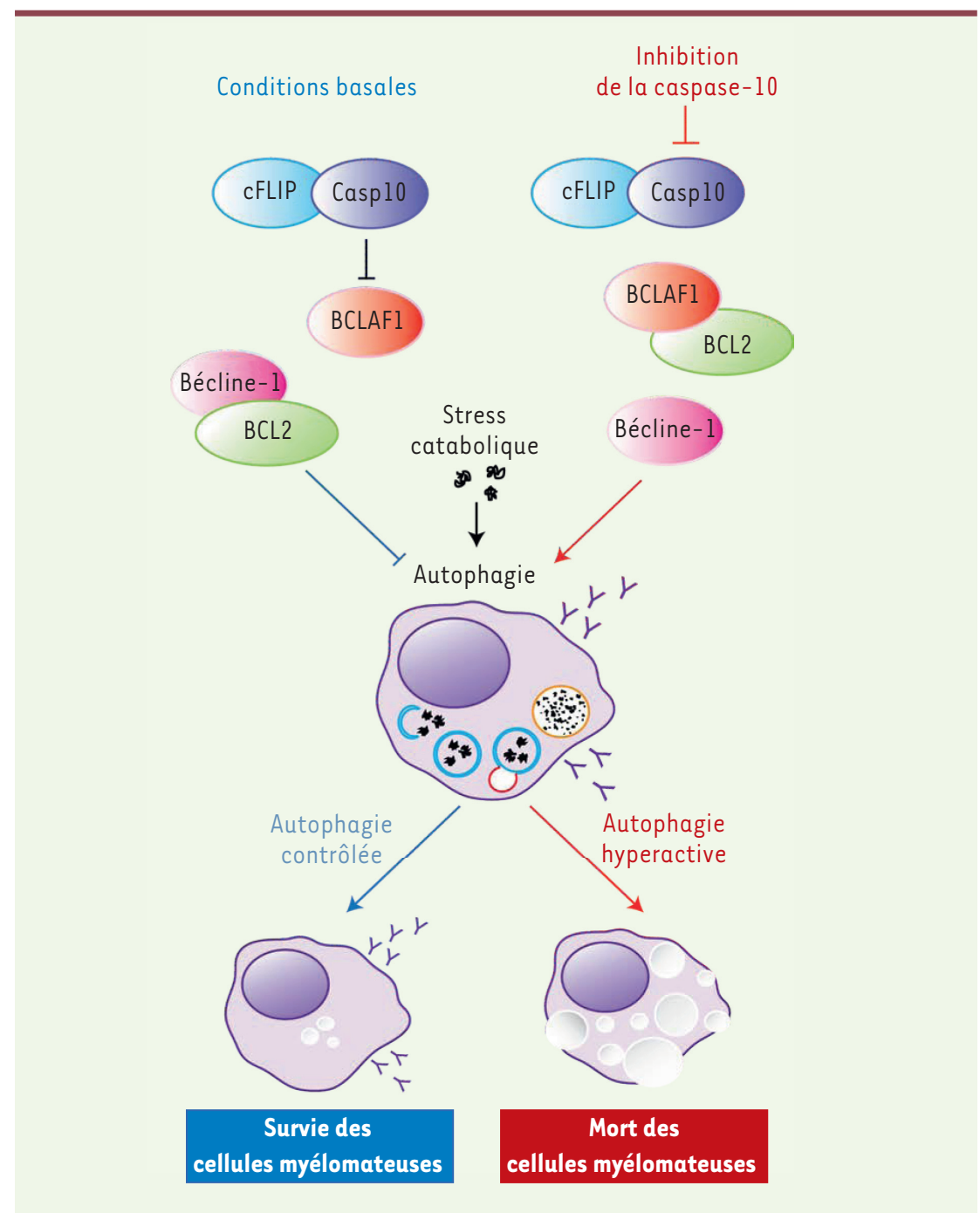

Figure 2. La survie des cellules de myélomes multiples est dépendante de la caspase-10. En conditions basales, la caspase- 10 partiellement active clive BCLAFl, empêchant ce dernier de s'associer à BCL2. Le complexe BCL2-bécline-1 reste stable, ce qui a pour effet de restreindre l'induction de l'autophagie et favorise la survie des myélomes multiples. En présence d'inhibiteurs de la caspase-10, BCLAFl n'est plus dégradée et s'accumule dans le cytoplasme. En s'associant à BCL2, elle déplace la liaison BCL2-bécline-1. La bécline-1 est alors libre de recruter les composants de la machinerie autophagique. Privée de son contrôle sur l'autophagie, la cellule s'engage dans un processus de mort cellulaire entretenu par une forme d'autophagie hyperactive. Casp 10 : caspase- 10 . autophagie hyperactive pourrait donc représenter une nouvelle perspective thérapeutique qui tire parti d'une vulnérabilité commune à tous ces types de cellules cancéreuses. $\diamond$

Multiple myeloma and autophagy

\section{LIENS D’INTÉRÊT}

L'auteur déclare n'avoir aucun lien d'intérêt concernant les données publiées dans cet article.

\section{RÉFÉRENCES}

1. Levine B, Kroemer $G$. Autophagy in the pathogenesis of disease. Cell $2008 ; 132: 27-42$.

2. Rabinowitz JD, White $\varepsilon$. Autophagy and metabolism. Science 2010 ; 330 : 1344-8.

3. Pengo N, Scolari M, Oliva L, et al. Plasma cells require autophagy for sustainable immunoglobulin production. Nat Immunol 2013 ; 14 : 298-305.

4. Mitsiades (S, Mitsiades NS, McMullan C), et al. Antimyeloma activity of heat shock protein-90 inhibition. Blood 2006 ; 107 : 1092-100.

5. Meister S, Schubert U, Neubert K, et al. Extensive immunoglobulin production sensitizes myeloma cells for proteasome inhibition. Cancer Res 2007 ; 67 : 1783-92.

6. Hoang B, Benavides A, Shi Y, et al. Effect of autophagy on multiple myeloma cell viability. Mol Cancer Ther $2009 ; 8$ : 1974-84.

7. Richardson PG, Mitsiades CS, Laubach JP, et al. Inhibition of heat shock protein 90 (HSP90) as a therapeutic strategy for the treatment of myeloma and other cancers. BrJ Haematol $2011 ; 152: 367-79$

8. Lamy L. Quand les cellules cancéreuses sont accros à un gène normal. Med Sci (Paris) $2009 ; 25: 9$-11.

9. Lamy L, Ngo VN, Emre NC, et al. Control of autophagic cell death by caspase-10 in multiple myeloma. Cancer Cell $2013 ; 23: 435-49$.

10. Kasof GM, Goyal L, White $\varepsilon$. Btf, a novel deathpromoting transcriptional repressor that interacts with Bcl-2-related proteins. Mol Cell Biol $1999 ; 19$ : 4390-404.

11. Pattingre S, Tassa A, Qu X, et al. Bcl-2 antiapoptotic proteins inhibit Beclin 1-dependent autophagy. Cell $2005 ; 122$ : 927-39.

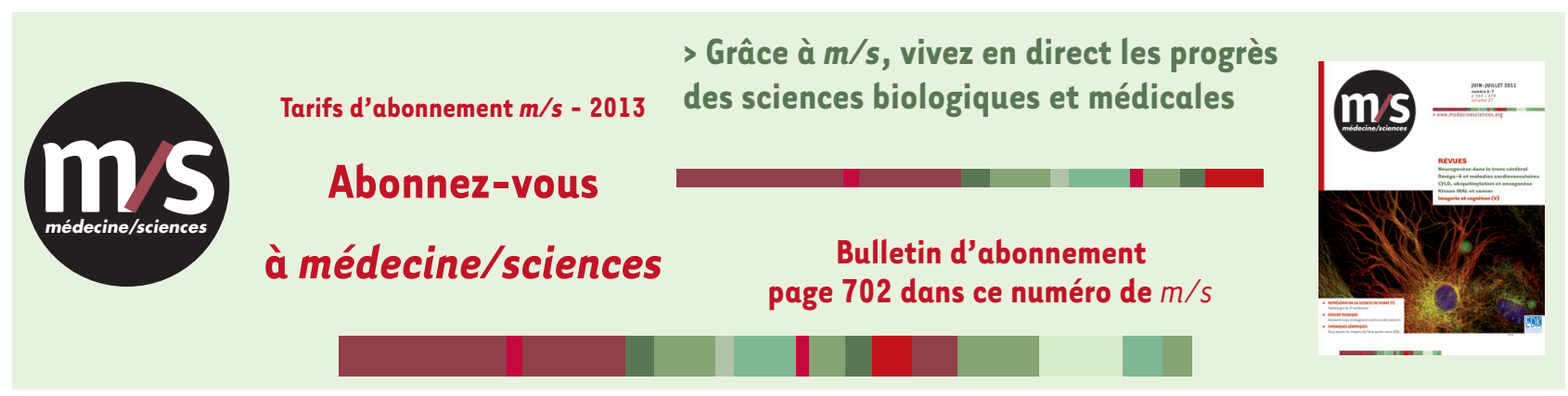

